\title{
Mechanical analysis of cranial distractor attachment with three different resorbable fixation systems
}

\author{
M. Savolainen ${ }^{\mathrm{a},}{ }^{*}$, A. Ritvanen ${ }^{\mathrm{b}}$, E. Tukiainen ${ }^{\mathrm{a}}$, J. Leikola ${ }^{\mathrm{a}}$ \\ ${ }^{a}$ Department of Plastic Surgery, Helsinki University Central Hospital, P.O. Box 266, 00290, HUS, Finland \\ b Department of Electrical Engineering and Automation, School of Electrical Engineering, Aalto University, P.0. Box 13000, 00076, Aalto, Finland
}

\section{A R T I C L E I N F O}

Article history:

Paper received 19 November 2017

Accepted 15 May 2018

Available online 21 May 2018

\section{Keywords:}

Posterior calvarial vault distraction

osteogenesis

Cranial distraction osteogenesis

Resorbable fixation

Ultrasound-activated pin

Heat-activated pin

Craniosynostosis

\begin{abstract}
A B S T R A C T
Distraction osteogenesis (DO) has become increasingly popular to correct craniosynostosis. Disadvantages of DO include the secondary operation needed for device removal and titanium screw related dura injury. To reduce invasiveness of the secondary device removal operation and to overcome titanium-related problems, fixation of the cranial distractor with resorbable materials is a potential alternative. New resorbable fixation methods, such as ultrasound-activated pins (UAPs) or heat-activated pins (HAPs), allow faster attachment on thinner bone than conventional resorbable screws (CRSs) since tapping is not required. However, resorbable materials are designed to be attached with a resorbable plate, not with a titanium distractor.

We evaluated the suitability of CRSs, HAPs and UAPs for the cranial distractor fixation in a laboratory setting with a mechanical testing machine. Fracture tests were conducted in two directions with respect to the longitudinal axis; vertical i.e. axial pull-out strength, and horizontal i.e. shear strength. Mean maximum pull-out strength for CRS, HAP and UAP was $48.9 \mathrm{~N}, 32.5 \mathrm{~N}$ and $14.7 \mathrm{~N}$, respectively. Mean maximum shear strength for CRS, HAP and UAP was $40.8 \mathrm{~N}, 77.9 \mathrm{~N}$ and $38.9 \mathrm{~N}$, respectively. According to our in vitro tests, the cranial distractor attachment with four CRSs or six HAPs per footplate would provide sufficient fixation stability.
\end{abstract}

() 2018 European Association for Cranio-Maxillo-Facial Surgery. Published by Elsevier Ltd. All rights reserved.

\section{Introduction}

Distraction osteogenesis (DO) has become increasingly popular to correct craniosynostosis. Advantages of posterior calvarial vault distraction osteogenesis (PCVO) relative to one-stage calvarial vault reconstruction (CVR) include a shorter, less invasive operation, good shape maintenance, greater potential for intracranial volume advancement and lower overall morbidity (Imai et al., 2002; Steinbacher et al., 2011; Lao and Denny, 2010; Kim et al., 2008). Gradual advancement ensures better soft tissue adaptation and better wound healing. DO represents a more physiological treatment than CVR since bone segment vascularity remains intact, no dead space is left between the dura and bone segments and no ossification defects remain (Nonaka et al., 2003; Lao and Denny, 2010). DO also provides cost savings (Ong et al., 2014).

\footnotetext{
* Corresponding author. Department of Plastic Surgery, Töölö Hospital, P.O. Box 266, FI-00290, HUS, Finland. Fax: +358 947187217

E-mail address: savolainen_mikko@yahoo.com (M. Savolainen).
}

A disadvantage of DO is the need for a second operation for device removal. In many cases, DO should be performed in early infancy to release increased intracranial pressure. However, the calvarium in early infancy may be too thin for distractors to be fixed with titanium screws (Steinbacher et al., 2011). The titanium screws might also injure the dura through thin bone (Nowinski et al., 2012).

To reduce the invasiveness of the secondary device removal operation, resorbable mandibular distractors were introduced. The same device has been used in PCVO (Maurice and Gachiani, 2014). The stability of the mandibular device in the calvarial region is questionable since resisting force in PCVO is reported to be higher than in mandibular DO and force-related complications, such as distractor breakage and footplate loosening, are commonly seen (Ritvanen et al., 2017; Imai et al., 2002; Nonaka et al., 2003; Steinbacher et al., 2011; White et al., 2008; Derderian et al., 2015; Nowinski et al., 2012).

The evolution of cranial distractors (Cranial vault distractor, KLS Martin, Germany) has resulted in increased strength of the device and positioning hooks on footplates being added that gain support on the bone edge and reduce the stress from screw fixation. In 
general, the attachment of a cranial distractor with resorbable materials is a potential option. New innovative resorbable fixation methods, such as ultrasound-activated pins (UAPs) and heatactivated pins (HAPs), allow attachment on thinner bone than with conventional resorbable screws (CRSs) since tapping is not required (Eckelt et al., 2007). Resorbable materials might also reduce the risk of titanium fixation-related dura injury through the thin bone.

Arnaud and Renier (2009) described fixation of cranial distractors with UAPs in monoblock DO. However, resorbable materials are designed to be attached with resorbable plates. UAPs fuse with the resorbable plate, increasing fixation strength (Pilling et al., 2007). Mechanical properties of resorbable fixation systems when attached via titanium distractor on bone remain unclear.

We designed a laboratory setting to evaluate the mechanical properties of three different resorbable fixation systems (CRS, HAP and UAP) when attached via titanium on bone. The aim was to find a safe resorbable fixation method to attach the distractor in calvarial DO.

\section{Materials and methods}

The porcine rib was chosen to most closely resemble the paediatric calvarial bone. The ribs were cut into $4 \mathrm{~cm}$ pieces from the same proximal area to provide homogeneous bone (Fig. 1). All soft tissue, including the periosteum, was meticulously dissected. The bones were split under the ventral cortex. The bone blocks were ground to a thickness of $4 \mathrm{~mm}$ to resemble the thickness of the paediatric calvarium (Fig. 1). The grinding was carefully orientated so that the ventral cortex and the dorsal surface were parallel (Fig. 1).

The universal testing machine (e3000LT, Instron, USA) was used to characterize the mechanical properties of resorbable fixation connected via a titanium plate to the bone. Two custom-made test set-ups were used to measure pull-out (Fig. 2-1) and shear (Fig. 2-2) strength on the testing machine.

We used 12 CRS $\left(1.5 \times 6 \mathrm{~mm}\right.$, Inion CPS ${ }^{\mathrm{TM}}$, Inion, Finland), 24:70:6 Poly (DL-Lactide-co-trimethylene carbonate); 12 HAP $(1.5 \times 6 \mathrm{~mm}$, Inion CMF RapidTack ${ }^{\mathrm{TM}}$, Inion, Finland), 17:78.5:4.5 Poly (DL-Lactideco-trimethylene carbonate); and $12 \mathrm{UAP}(1.6 \times 5 \mathrm{~mm}$, Sonic Weld, KLS Martin, Germany), 50:50 poly (DL-lactide). We used in both shear and pull-out set-ups 6 CRSs, 6 HAPs and 6 UAPs. The screws and pins were connected to bone via the titanium plate (2.3 $\mathrm{mm}$ Smart Shape plate, KLS Martin, Germany). In the pull-out set-up, titanium plates were bent from both sides of the hole, where resorbable material was inserted at an angle of $90^{\circ}$ (Figs. 2-1 and 3-2).

The screws and pins were individually fixed with the titanium plate on the outermost point, i.e. tangential to the concave-shaped

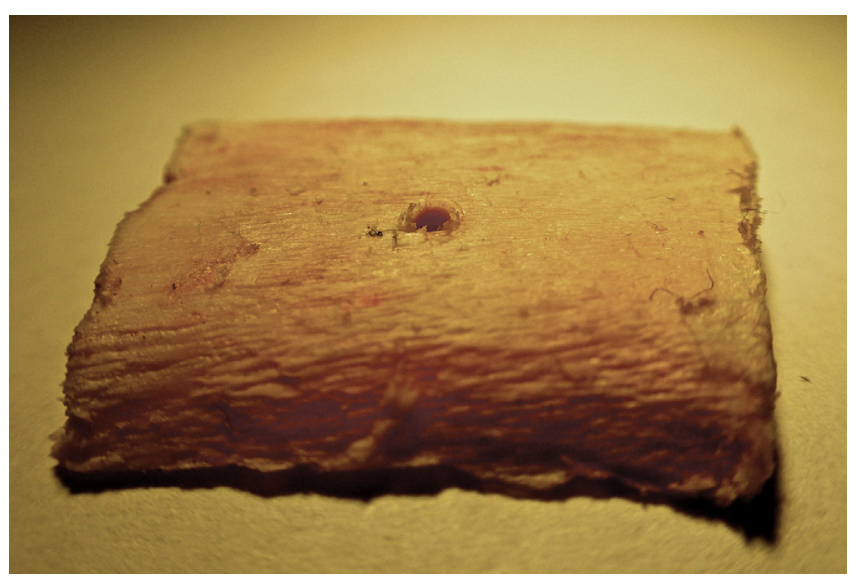

Fig. 1. Porcine rib prepared for the test set-up. rib, as in clinical situations with dedicated tools. Fixation was done by a person familiar with all of the fixation devices and according to manufacturers' instructions.

Tapping for the CRSs was accomplished with the dedicated selftapping thread $1.5 \times 8 \mathrm{~mm}$. The screws were inserted to the bone through the titanium plate with the dedicated screw driver. The holes for the HAPs were drilled by the dedicated thread $1.6 \times 8 \mathrm{~mm}$. The HAPs were inserted to the bone through the titanium plate, and heat activation with a dedicated tool was performed until the pin was attached. Holes were drilled for UAPs with the $1.0 \times 4 \mathrm{~mm}$ thread. The UAPs were inserted into the drilled hole through the titanium plate. Ultrasound activation by a sonotrode was provided until the pin had reached the bottom of the hole.

All test samples were set in an incubation bath $\left(\mathrm{NaCl} 0.9 \%, 26{ }^{\circ} \mathrm{C}\right)$ for $20 \mathrm{~h}$. After the incubation, the test samples were kept inside moist paper at room temperature $\left(26^{\circ} \mathrm{C}\right)$ for $30 \mathrm{~min}$ to $3 \mathrm{~h}$ before testing.

The titanium plate and the bone were connected to a testing machine with the designed pull-out (Fig. 2-1) and shear (Fig. 2-2) set-ups. The fixation was always orientated so that the force generated by the testing machine was conducted at a $90^{\circ}$ angle to the fixation. The bone segments were firmly fixed to the testing set-ups, paying special attention to the tensile force direction to remove any backlash in the set-up. The testing machine conducted force to the fixation at a speed of $0.05 \mathrm{~mm} / \mathrm{s}$ until breakage. The force conducted by the testing machine and the displacement was recorded at an amplitude of $50 \mathrm{~Hz}$.

The maximum force $(\mathrm{N})$ and displacement $(\mathrm{mm})$ for each test were recorded. Also elongation $(\mathrm{mm})$ at the break was recorded. The energy $(\mathrm{mJ})$ needed to break the fixations was calculated from the force displacement curve for each test. Energy needed to reach maximum force was used, rather than total energy in the tests, as after the force required reaching further displacement starts to decrease, the fixation can be considered to have failed.

\subsection{Statistical methods}

Means and standard deviations were determined for each test group. Two-tailed Student's $t$-test was used to calculate statistical significance between the groups ( $p$-value). A $p$-value of 0.05 was considered statistically significant. Excel software (Excel, Microsoft, USA) was used for data processing.

\section{Results}

Thirty-five fracture tests were performed: 12 for CRS, 12 for HAP and 11 for UAP. The results are presented in Table 1. One UAP pullout test was abandoned due to test sample breakage before testing. One HAP pull-out test was abandoned because of technical failure. Thus, five pull-out tests were included in the study for HAP and UAP.

\subsection{Pull-out test}

The mean maximum strength was for CRSs $48.9 \mathrm{~N}$ (SD 8.4, Range 37.8-60.0), for HAPs $32.5 \mathrm{~N}$ (SD 16.2 Range 18.3-53.0) and for UAPs $14.5 \mathrm{~N}$ (SD 7.5, Range 5.6-24.0). The results between CRSs and UAPs were statistically significant ( $p$-0.000006).

The mean energy needed to break the fixation was for CRSs $9.0 \mathrm{~mJ}$ (SD 3.4, Range 4.9-12.9), for HAPs $21.7 \mathrm{~mJ}$ (SD 36.5, Range 1.7-86.3) and for UAPs $1.3 \mathrm{~mJ}$ (SD 0.8, Range 0.5-2.2). The results between CRSs and UAPs were statistically significant $(p-0.002)$.

The mean elongation at the break was for CRSs $0.03 \mathrm{~mm}$ (SD 0.09 , Range $0.2-0.4$ ), for HAPs $0.7 \mathrm{~mm}$ (SD 0.8, Range 0.2-2.2) and for UAPs $0.2 \mathrm{~mm}$ (SD 0.1, Range 0.09-0.4). The results were not statistically significant between the groups. 


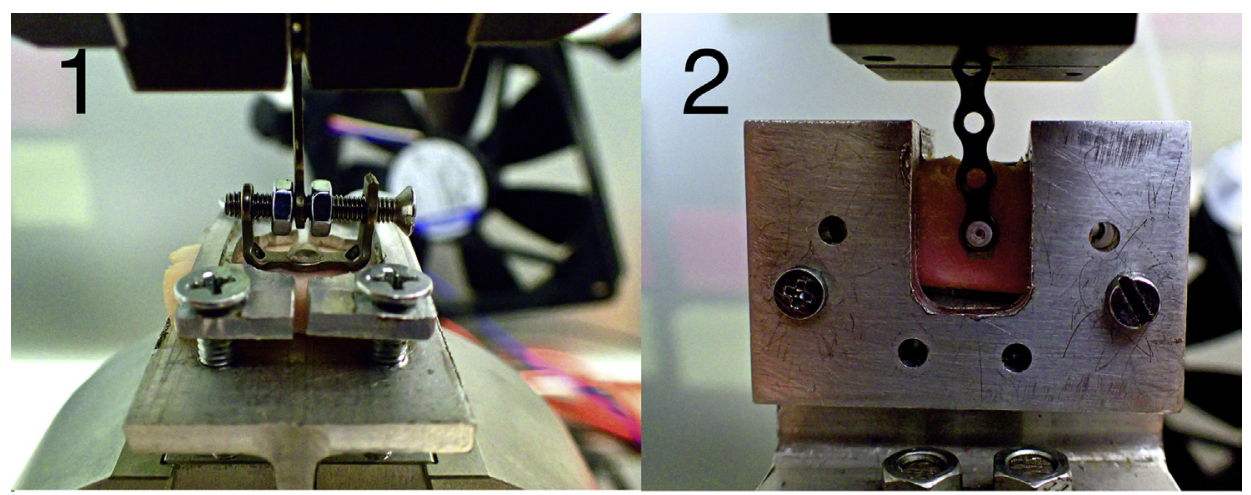

Fig. 2. Custom-made test set-ups. 2-1 pull-out set-up. 2-2 shear set-up.

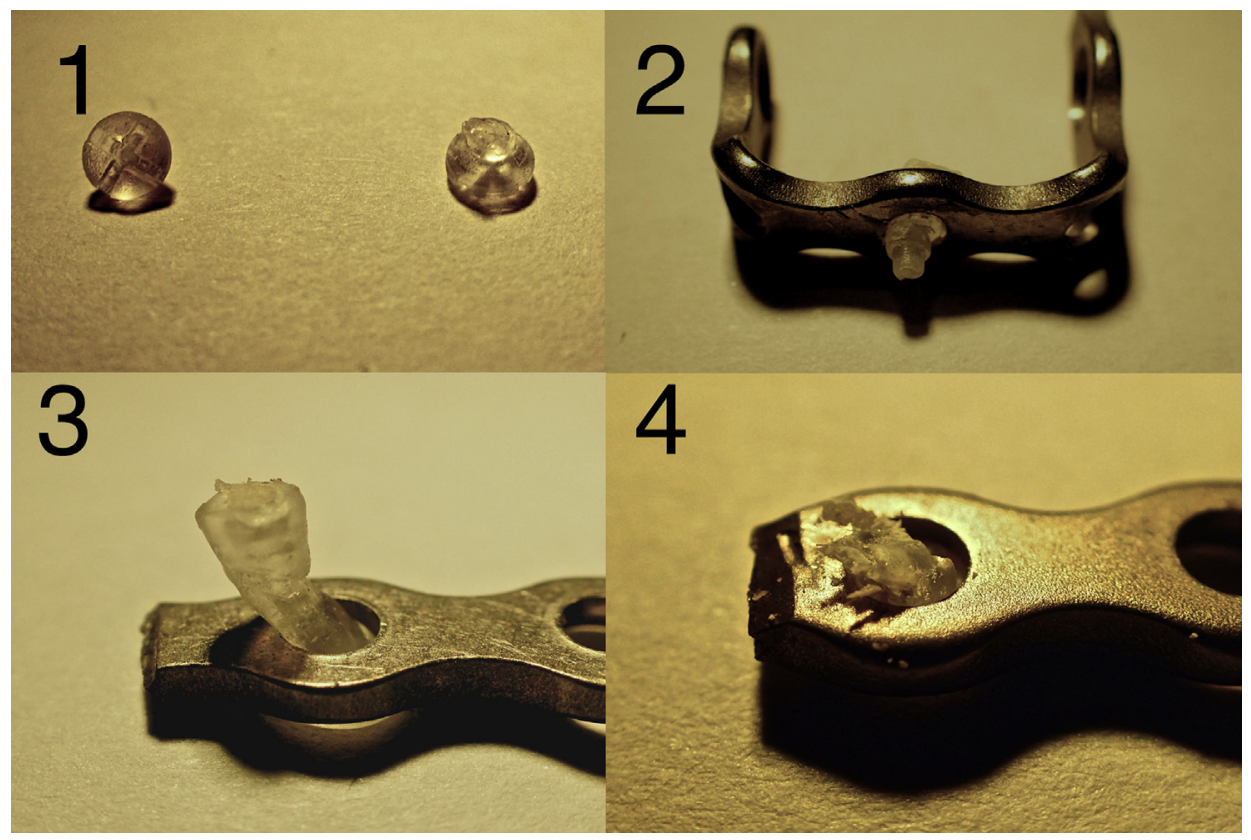

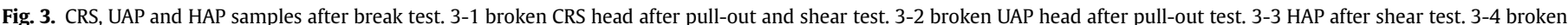
UAP after shear test.

Table 1

The fracture test results for conventional resorbabla screws (CRS), heat activated pins (HAP) and utrasound activated pins (UAP).

\begin{tabular}{|c|c|c|c|}
\hline Pull-out Test & Mean strength $(\mathrm{N})$ & Energy to break (mJ) & Elongation at break (mm) \\
\hline CRS & $48.9(\operatorname{SD} 8.4, \mathrm{n}=6)$ & 9.0 (SD 3.4) & 0.3 (SD 0.09) \\
\hline HAP & $32.5($ SD $16.2, \mathrm{n}=5)$ & $21.7(36.5)$ & 0.7 (SD 0.8) \\
\hline UAP & $14.7(\mathrm{SD} 7.5, \mathrm{n}=5)$ & $1.3(\mathrm{SD} 0.8)$ & $0.2(\mathrm{SD} 0.1)$ \\
\hline Shear Test & Mean strength $(\mathrm{N})$ & Energy to break (mJ) & Elongation at break ( $\mathrm{mm})$ \\
\hline CRS & $40.8(\operatorname{SD} 6.0, \mathrm{n}=6)$ & $31.8($ SD 8.2$)$ & $1.3(\mathrm{SD} 0.3)$ \\
\hline HAP & $77.9($ SD $18.3, \mathrm{n}=6)$ & $141.3($ SD 91.5$)$ & $2.3(\mathrm{SD} 0.1)$ \\
\hline UAP & $38.9(\mathrm{SD} 14.9, \mathrm{n}=6)$ & $20.3($ SD 12.9) & 0.7 (SD 0.3) \\
\hline
\end{tabular}

Five CRS shafts broke underneath the titanium plate and one screw pulled out from the bone without breakage (Fig. 3-1). The CRS fracture surfaces showed characteristics of tough fractures with plastic deformation.

Five HAPs were pulled out without breakage but with slight plastic deformation. Four UAPs broke underneath the titanium plate (Fig. 3-2) and one was pulled out without breakage. The UAP fracture surfaces showed characteristics of tough fractures with plastic deformation.

\subsection{Shear test}

The mean maximum strength was for CRSs $40.8 \mathrm{~N}$ (SD 6.0, Range 31.7-47.1), for HAPs 77.9 N (SD 18.3, Range 52.0-95.4) and for UAPs 38.9 (SD 14.9, Range 17.8-58.1). The results were statistically significant between HAPs and CRSs $(p-0.0031)$ and between HAPs and UAPs ( $p-0.0031)$.

The mean energy needed to break the fixation was for CRSs $31.8 \mathrm{~mJ}$ (SD 8.2, Range 14.8-41.2), for HAPs $141.3 \mathrm{~mJ}$ (SD 91.5, Range 
42.6-251.1) and for UAPs $20.3 \mathrm{~mJ}$ (SD 12.9, Range 6.2-42.7). The results were statistically significant between HAPs and CRSs and UAPs ( $p-0.03)$ and between UAPs and HAPs ( $p-0.04)$.

The mean elongation at the break was for CRSs $1.3 \mathrm{~mm}$ (SD 0.3, Range 0.7-1.6), for HAPs $2.3 \mathrm{~mm}$ (SD 1.0, Range 0.9-3.4 N) and for UAPs $0.7 \mathrm{~mm}$ (SD 0.3, Range 0.4-1.0). The results were statistically significant between CRSs and UAPs $(p-0.008)$ and between HAPs and UAPs $(p-0.01)$.

Six CRS shafts broke underneath the titanium plate (Fig. 3-1). The CRS fracture surfaces showed characteristics of tough fractures with plastic deformation. Six HAPs were pulled out from the bone without breakage but with plastic deformation (Fig. 3-3). Six UAPs broke underneath the titanium plate (Fig. 3-4). The UAP fracture surfaces showed characteristics of tough fractures with plastic deformation.

\subsection{Case report}

A seven-month-old girl with Crouzon syndrome and bilateral coronal synostosis was treated with PCVO at Helsinki University Hospital. A bicoronal zic-zac incision was made to access calvaria. Bicoronal osteotomy and horizontal osteotomy above the inion were performed to create a maximal free floating posterior bone flap. Four cranial distractors were positioned between the osteotomy lines (Fig. 4). Distraction vectors were orientated parallel antero-posteriorly. Footplate positioning hooks were carefully orientated to gain support from the bone edges. Each footplate was attached with four conventional resorbable screws $(1.5 \mathrm{~mm}$, Rapidsorb, Synthes, USA), 85:15 poly (L-lactide-co-glycolide). Distractors were activated and a $2 \mathrm{~mm}$ gap was left between the osteotomy lines. A latency period of 5 days was used. The distraction rate was $1.2 \mathrm{~mm}$ once daily, and a total distraction distance of $20 \mathrm{~mm}$ was achieved. Distractors were removed in a second operation after a 1-month stabilization phase. Bicoronal incision was performed on the old scar. The distractors could be removed by bending the device from the distractor arms. Secondary frontoorbital advancement was performed at the age of 15 months. No complications occurred during the treatment.

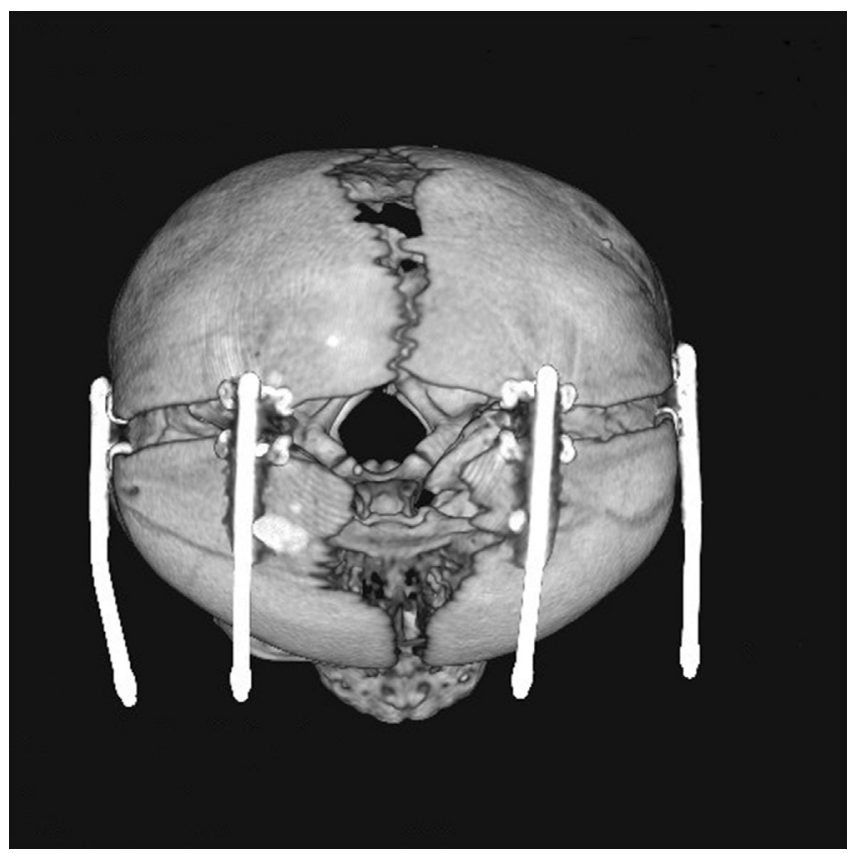

Fig. 4. Four cranial distractors of a PCVO patient were attached with conventional resorbable screws.

\section{Discussion}

This study explores the mechanical properties of three different resorbable fixation systems connected via titanium to bone. The peak force recorded in PCVO was $74 \mathrm{~N}$ for patients younger than 13 months (Ritvanen et al., 2017). Thus, footplate fixation with four CRSs and six HAPs provide $200 \%$ of the required fixation strength in the pull-out and shear directions. Our earlier study showed that the CRS in clinical use has comparable strength to the tested CRS (Savolainen et al., 2015). Attachment of the cranial distractor with four CRSs per footplate provided sufficient fixation strength in our PCVO clinical case.

Force is directed perpendicularly with respect to the distraction vectors and is conducted via shear strength to the fixation material. The cranial distractor positioning hooks can reduce the shear stress from the fixation by supporting the bone edges. In any case, torque force can be conducted to the fixation. Both shear and pull-out fixation strengths are relevant in calvarial DO.

The UAPs are designed to fuse with the resorbable plate, increasing the strength of the fixation (Pilling et al., 2007). The weakest and narrowest point of the UAPs is the neck; fusion with the resorbable plate is likely to increase the strength of the weakest point (Savolainen et al., 2015). The UAP fixation with the resorbable plate as designed probably explains the earlier observed high stability (Pilling et al., 2007). However, attaching the cranial distractor with UAPs has already been found successful in a clinical setting (Arnaud and Renier, 2009). The aim of this study was to provide further information about cranial distractor attachment in a laboratory setting. An in vitro model cannot fully match in vivo conditions. Therefore more research is needed in order to reach a final conclusion about cranial distractor attachment with different resorbable fixation systems.

The energy needed to break the fixation illustrates the toughness of the material. Tougher material allows more elongation, providing a more stable fixation on the concave-shaped calvarium. Tougher material is also more stable due to shock absorption. Traumas have been reported to cause footplate loosening (Steinbacher et al., 2011). Thus, toughness is an important factor when mechanical properties of a fixation material are evaluated.

High toughness was observed for HAPs, especially in the shear direction. HAPs are produced by a self-reinforcement technique. Implants produced by this technique exhibit high orientation of polymer fibres, increasing the strength along their long axis (Törmälä, 1992). Higher toughness makes HAPs a potential option for distractor fixation.

Most CRSs and UAPs broke at the shaft underneath the titanium plates, indicating that the material-bone interface is not a limiting factor for fixation strength. The higher variation in the fixation strength for HAPs than for CRSs or UAPs can be explained by pin-bone interface stability since HAPs were pulled out from the bone without material breakage (Fig. 3-3). The device needed to heat, i.e. activate, the HAPs is a prototype of the product coming to market. Thus, these results might not be entirely accurate for the upcoming product.

A drawback of DO is the second operation needed to remove the distractors (Derderian and Seaward, 2012). To address this issue, resorbable mandibular distractors were introduced, which can be removed through the exit hole of the distractor arm. The same device was used in PCVO (Maurice and Gachiani, 2014). The resisting force in calvarial DO is higher than in mandibular DO (Ritvanen et al., 2017). Thus, the mandibular device stability is questionable since distractor breakage and footplate loosening are commonly seen in calvarial DO (Imai et al., 2002; Nonaka et al., 2003; Steinbacher et al., 2011; White et al., 2008; Derderian et al., 2015; Nowinski et al., 2012; Ong et al., 2014). Fixation of a more 
stable cranial distractor with resorbable materials can thus be a better option.

The CRSs and HAPs lose about $52-70 \%$ of their strength between 12 and 26 weeks after the implantation (Nieminen et al., 2007). However, the self-reinforcement of HAPs can increase degradation time (Tormala, 1992). The UAPs should provide strength of $80 \%$ and $55 \%$ two and six months after implantation, respectively (Nguyen et al., 2017). The resorption profile of all tested materials should provide sufficient strength for the consolidation, distraction and stabilization phases. The weakening of the material could allow removal of the cranial distractor through the distractor arm exit after the stabilization phase (2-5 months after implantation), although no data are available on the actual resorption rate of these materials in clinical situations. In our case report, bending from the distractor arms could fracture the distractor fixation in the device removal operation. That indicates that the cranial distractor fixed with resorbable material could be removed through the distractor arm exit. However, the removal operation was easier and less invasive in our case than with titanium fixation.

The paediatric calvarium is often too thin for footplate attachment using conventional screws. Thus, PCVO is usually performed on patients older than 6 months when the bone is thicker (Nonaka et al., 2003; Steinbacher et al., 2011). In some cases, resolving an ICH is necessary before the age of 6 months. Fixation can be done on the thinner bone with HAPs since tapping is not required (Eckelt et al., 2007). Another benefit lies in the use of resorbable fixation screws or pins, which can reduce the risk of a fixation-related dura injury through the thin bone.

No data are yet available about the resisting force in PCVO for patients older than 13 months. The resisting force might be higher for these patients due to a thicker callus and scalp. This assumption is supported by distractor breakage being observed especially in older patients (Ong et al., 2014). Also missing are data on resisting forces for other types of calvarial DO besides PCVO. In monoblock $\mathrm{DO}$, the resisting force is probably similar to that in PCVO according to Newton's third law. Suitability of resorbable distractor fixation for patients older than 13 months and for other types of calvarial DO remains unclear.

The same fixation stability cannot be achieved with resorbable materials as with titanium. Thus, any additional stress from the fixation should be avoided. An earlier study showed that performing distraction in a more gradual manner would decrease the distraction force (Ritvanen et al., 2017). Accurate vector positioning is imperative to avoid conflicting distraction vectors and torque forces from the attachment and to maintain the cranial distractors position hook support from the bone edges.

Continuous force monitoring during DO would allow adjusting the distraction rate to avoid fixation or device breakage. Moreover, a desynchronous force increase between the distractors could be a sign of conflicting distraction vectors or another device-related problem. Some of these complications might be avoided if the problem was recognized sufficiently early.

Earlier studies on resorbable materials have used red oak wood, sheep bone and polymethylmethacrylate as ground material for fixation (Pilling et al., 2007; Buijs et al., 2009). Porcine rib is softer than the human cadaveric mandible (Bredbenner and Haug, 2000). The paediatric neurocranium is characterized as a soft and viscoelastic structure (Margulies and Thibault, 2000). We therefore consider porcine rib to have the closest substitutional biomechanical and structural properties to the paediatric neurocranium. Natural bone could represent a better material-bone interface than synthetic materials.

We do not know the actual resorption rate of the tested materials in a clinical situation. Thus, the fixation stability and the possibility of removing the distracters through the distractor arm exit warrant further research. In addition, the suitability of resorbable distractor fixation for older patients ( $>13$ months) and for types of calvarial DO other than PCVO or monoblock remains to be elucidated. Clinical studies are needed to provide answers for these questions.

\section{Conclusions}

According to our in vitro tests, attaching a cranial of distractor with four CRSs or six HAPs per footplate can provide sufficient stability in PCVO and monoblock DO. The resorbable fixation reduces invasiveness of the secondary device removal operation and might reduce the titanium fixation-related dura injury. Fixation with HAPs allows fixation on thinner bone since tapping is not required.

\section{Sources of support in the form of grants}

No financial support was received for this study.

\section{Declaration of interests}

No conflicts of interest.

\section{Acknowledgements} study.

Authors acknowledge Synoste for technical support to this

\section{References}

Arnaud E, Renier D: Pediatric craniofacial osteosynthesis and distraction using an ultrasonic-assisted pinned resorbable system: a prospective report with a minimum 30 months' follow-up. J Craniofac Surg 20(6): 2081-2086, 2009

Bredbenner T, Haug R: Substitutes for human cadaveric bone in maxillofacial rigid fixation research. Oral Surg Oral Med Oral Pathol Oral Radiol Endod 90(5): 574-580, 2000

Buijs G, van der Houwen E, Stegenga B, Verkerke G, Bos R: Mechanical strength and stiffness of the biodegradable SonicWeld Rx osteofixation system. J Oral Maxillofac Surg 67(4): 782-787, 2009

Derderian C, Wink J, McGrath J, Collinsworth A, Bartlett S, Taylor J: Volumetric changes in cranial vault expansion: comparison of fronto-orbital advancement and posterior cranial vault distraction osteogenesis. Plast Reconstr Surg 135(6): 1665-1672, 2015

Derderian C, Seaward J: Syndromic craniosynostosis. Semin Plast Surg 26(02): 064-075, 2012

Eckelt U, Nitsche M, Muller A, Pilling E, Pinzer T, Roesner D: Ultrasound aided pin fixation of biodegradable osteosynthetic materials in cranioplasty for infants with craniosynostosis. J Craniomaxillofac Surg 35(4-5): 218-221, 2007

Imai K, Komune H, Toda C, Nomachi T, Enoki E, Sakamoto H, et al: Fujimoto: cranial remodeling to treat craniosynostosis by gradual distraction using a new device. J Neurosurg 96(4): 654-659, 2002

Kim Y, Choi J, Kim D, Lee W, Yoo S, Kim H, et al: Cranial growth after distraction osteogenesis of the craniosynostosis. J Craniofac Surg 19(1): 45-55, 2008

Lao W, Denny A: Internal distraction osteogenesis to correct symptomatic cephalocranial disproportion. Plast Reconstr Surg 126(5): 1677-1688, 2010

Margulies S, Thibault K: Infant skull and suture properties: measurements and implications for mechanisms of pediatric brain injury. J Biomech Eng 122(4): 364-371, 2000

Maurice S, Gachiani J: Posterior cranial vault distraction with resorbable distraction devices. J Craniofac Surg 25(4): 1249-1251, 2014

Nguyen D, Woo A, Farber S, Skolnick G, Yu J, Naidoo S, et al: Comparison of resorbable plating systems. J Craniofac Surg 28(1): 88-92, 2017

Nieminen T, Rantala I, Hiidenheimo I, Keränen J, Kainulainen H, Wuolijoki E, et al: Degradative and mechanical properties of a novel resorbable plating system during a 3-year follow-up in vivo and in vitro. J Mater Sci Mater Med 19(3): 1155-1163, 2007

Nonaka Y, Oi S, Miyawaki T, Shinoda A, Kurihara K: Indication for and surgical outcomes of the distraction method in various types of craniosynostosis. Childs Nerv Syst 20(10): 702-709, 2003

Nowinski D, Di Rocco F, Renier D, SainteRose C, Leikola J, Arnaud E: Posterior cranial vault expansion in the treatment of craniosynostosis. Comparison of current techniques. Childs Nerv Syst 28(9): 1537-1544, 2012

Ong J, Harshbarger R, Kelley P, George T: Posterior cranial vault distraction osteogenesis: evolution of technique. Semin Plast Surg 28(4): 163-178, 2014 
Pilling E, Meissner H, Jung R, Koch R, Loukota R, Mai R, et al: An experimental study of the biomechanical stability of ultrasound-activated pinned (SonicWeld $\mathrm{Rx}^{(}+$Resorb- $\mathrm{X}^{\mathbb{R}}$ ) and screwed fixed (Resorb- $\mathrm{X}^{\circledR}$ ) resorbable materials for osteosynthesis in the treatment of simulated craniosynostosis in sheep. Br J Oral Maxillofac Surg 45(6): 451-456. https://doi.org/10.1016/ j.bjoms.2006.12.008, 2007

Ritvanen A, Savolainen M, Novinski D, Saiepour D, Paulasto-Kröckel M, Hukki J, et al: Force measurements during posterior calvarial vault osteodistraction: a novel measurement method. J Craniomaxillofac Surg 45(6): 981-989, 2017
Savolainen M, Ritvanen A, Koljonen V, Turunen M, Hulkkonen H, Vuorinen V, et al: Mechanical analysis of ultrasound-activated pins and resorbable screws. J Craniofac Surg 26(4): 1234-1237, 2015

Steinbacher D, Skirpan J, Puchała J, Bartlett S: Expansion of the posterior crania vault using distraction osteogenesis. Plast Reconstr Surg 127(2): 792-801, 2011

Törmälä P: Biodegradable self-reinforced composite materials; manufacturing structure and mechanical properties. Clin Mater 10(1-2): 29-34, 1992

White N, Evans M, Dover M, Noons P, Solanki G, Nishikawa H: Posterior calvarial vault expansion using distraction osteogenesis. Childs Nerv Syst 25(2): 231-236, 2008 